\title{
THE FUNDAMENTAL GROUP OF A COMPACT FLAT LORENTZ SPACE FORM IS VIRTUALLY POLYCYCLIC
}

\author{
WILLIAM M. GOLDMAN \& YOSHINOBU KAMISHIMA
}

A flat Lorentz space form is a geodesically complete Lorentzian manifold of zero curvature. It is well known (see Auslander \& Markus [3]) that such a space $M$ may be represented as a quotient $\mathbf{R}^{n} / \Gamma$, where $\mathbf{R}^{n}$ is an $n$-dimensional Minkowski space ( $n$ equals the dimension of $M$ ) and $\Gamma$ is a group of Lorentz isometries acting properly discontinuously and freely on $\mathbf{R}^{n}$. In particular the universal covering of $M$ is isometric to $\mathbf{R}^{n}$ and the fundamental group $\pi_{1}(M)$ is isomorphic to $\Gamma$.

Theorem. Let $M$ be a compact flat Lorentz space form. Then $\pi_{1}(M)$ is virtually polycyclic.

Recall that a group is virtually polycyclic if it can be built by iterated extensions from finitely many finite groups and cyclic groups. This result affirms a conjecture of Milnor [13] in a special case. For discussion of this conjecture as well as another special case, we refer to Fried \& Goldman [8].

The importance of this result is that it reduces the classification of compact flat Lorentz space forms to fairly elementary problems concerning Lie algebras and lattices in solvable Lie groups; we hope to pursue this classification in a future publication. For a description of this reduction and the classification in dimension 3, see Fried \& Goldman [8]; in dimension 4 the classification is worked out in Fried [7]. One immediate consequence of the structure theory developed in Fried \& Goldman [8, §1] and Kamishima [12] is the following

Corollary. Let $M$ be the compact flat Lorentz space form. Then $M$ has a finite covering which is diffeomorphic to a solvmanifold.

The outline of this paper is as follows. In the first section we collect some basic facts about the group $E(n-1,1)$ of isometries of $n$-dimensional Minkowski space. In the second section we prove the theorem in the special

Received December 14, 1982, and, in revised form, September 3, 1983. The research was supported in part by NSF Grant MCS-8202082. 
case that the linear holonomy group of $M$ is discrete. Finally, in the third section we remove this discreteness assumption, thereby concluding the proof of the theorem.

\section{Some algebraic lemmas}

1.1. We give $\mathbf{R}^{n}$ with the Lorentzian inner product $\omega=d x_{1}^{2}+\cdots+d x_{n-1}^{2}$ $-d x_{n}^{2}$. Denote by $E(n-1,1)$ the group of all Lorentz isometries $\mathbf{R}^{n} \rightarrow \mathbf{R}^{n}$. It is well known that $E(n-1,1)$ is a semidirect product, $T \rtimes O(n-1,1)$ is where $T \cong \mathbf{R}^{n}$ denotes the group of translations and $O(n-1,1)$ is the homogeneous Lorentz group (orthogonal group) consisting of all linear mappings preserving $\omega$. Let $L: E(n-1,1) \rightarrow O(n-1,1)$ denote the projection homomorphism with kernel $T$. Let $E^{0}(n-1,1)$ denote the identity component of $E(n-1,1)$; then $E^{0}(n-1,1)$ is the semidirect product $T \times S O_{+}(n-1,1)$ where $S O_{+}(n-1,1)$ is the identity component of $O(n-1,1)$ (consisting of orientation-preserving, causality-preserving Lorentz-orthogonal transformation).

A group is virtually polycyclic if it has a polycyclic subgroup of finite index. It is well known (see Milnor [3, 2.2]) that a discrete subgroup $\Gamma$ of a connected Lie group which contains a solvable subgroup of finite index is virtually polycyclic. Recall that a connected Lie group $G$ is amenable if it splits as a semidirect product $S \rtimes K$, where $S$ is a solvable normal subgroup and $K$ is compact. Then it is also well known that a discrete subgroup of a connected amenable Lie group $G$ is virtually polycyclic (Milnor [13, 2.2]). Thus, if $\Gamma \subset E(n-1,1)$ is a discrete subgroup and $L(\Gamma)$ lies in an amenable subgroup of $O(n-1,1)$ which has finitely many components, then $\Gamma$ is virtually polycyclic.

1.2. In order to apply this observation, we must understand the structure of the connected subgroups of $O(n-1,1)$. A thorough discussion is given in Greenberg [10, §4] as well as Chen \& Greenberg [6, §4].

Let $V \subset E$ be a linear subspace of dimension $k<n$ and let $G_{V}$ be its stabilizer. Then the quadratic form on $\mathbf{R}^{n}$ defining $O(n-1,1)$ restricts to a quadratic form $q_{V}$ on $V$ which may be positive definite, indefinite (or negative definite), or degenerate. If $q_{V}$ is positive, then there exists $q \in O(n-1,1)$ such that $g V=\mathbf{R}^{k} \times\{0\} \subset \mathbf{R}^{n}$ and $G_{V}$ is conjugate by $g$ to the subgroup $O(k) \times$ $O(n-k-1,1)$ of $O(n-1,1)$. In case $q_{V}$ is indefinite (or negative definite if $k=1)$, then $V$ is equivalent to subspace $\{0\} \times \mathbf{R}^{k} \subset \mathbf{R}^{n}$ and $G_{V}$ is conjugate to $O(n-k) \times O(k-1,1) \subset O(n-1,1)$. If $q_{V}$ is degenerate, then the kernel of $q_{V}$ consists of all null vectors in $V$ and is one-dimensional. It is easy to see that the stabilizer of a null ray is an amenable Lie group (it is isomorphic to the 
group of Euclidean similarities $\left.\mathbf{R}^{n-2} \rtimes\left(\mathbf{R}^{*} \times S O(n-2)\right)\right)$. Thus, it follows from 1.1 that if $\Gamma \subset E(n-1,1)$ is a discrete subgroup such that $L(\Gamma)$ normalizes a linear subspace of $\mathbf{R}^{n}$ upon which the Lorentzian metric is degenerate, then $\Gamma$ is virtually polycyclic.

1.3. These ideas play a key role in the proof of the following lemma, which although known, is difficult to find stated in the literature.

Lemma. Let $G \subset O(n-1,1)$ be a nontrivial closed connected amenable (e.g. solvable) subgroup of $O(n-1,1)$ and $N(G)$ its normalizer. Then:

(a) If $G$ is noncompact, then $N(G)$ is amenable.

(b) If $G$ is compact, then either $N(G)$ is compact or there exists $k, 1 \leqslant k \leqslant$ $n-1$, and $a g \in O(n-1,1)$ such that

$$
g^{-1} N(G) g \subset O(k) \times O(n-k-1,1) \subset O(n-1,1)
$$

and

$$
g^{-1} G g \subset O(k) \times\{1\} \subset O(n-1,1) .
$$

Proof. Let $q\left(x_{1}, \cdots, x_{n}\right)=x_{1}^{2}+\cdots+x_{n-1}^{2}-x_{n}^{2}$ be the quadratic form invariant under $O(n-1,1)$ and let $L=\left\{x \in \mathbf{R}^{n}: x_{n}>0, q(x)<0\right\}$ be the positive half of the light cone, $\bar{L}$ its closure and $\partial L=q^{-1}(0) \cap\left\{x_{n} \geqslant 0\right\}$ its boundary. Since $G$ is amenable it preserves at least one ray in the compact convex cone $\bar{L}$ (Greenleaf [10]). Let $\Lambda$ be the set of all such rays and $\Sigma$ their linear span in $\mathbf{R}^{n}$. Clearly $\Lambda$ and $\Sigma$ are invariant under $N(G)$.

We claim that $\Sigma \neq \mathbf{R}^{n}$. We may assume $n \geqslant 2$ (the case $n=2$ can be handled separately.) For if $\Sigma=\mathbf{R}^{n}$ there would exist $n$ linearly independent vectors $e_{1}, \cdots, e_{n} \in \bar{L}$ which would be eigenvalues for all of the elements of $G$. For each $j=1, \cdots, n$ the orthogonal complement of the span of $e_{1}, \cdots, e_{j-1}$, $e_{j+1}, \cdots, e_{n}$ is a line disjoint from $L \backslash\{0\}$ and contains a vector $v_{j}$ with $q\left(v_{j}\right)=+1$. Clearly $\left\{V_{1}, \cdots, V_{j}\right\}$ is a linearly independent set of simultaneous eigenvectors for $G$ and for each $g \in G, g v_{i}=v_{i}$ since $g$ preserves $q$ and $q\left(v_{j}\right)=+1$. Thus $G$ fixes a basis of $\mathbf{R}^{n}$ and must be trivial, a contradiction.

Now we prove (a). Suppose $G$ is noncompact. Then $\Lambda \subset \partial L$. For otherwise $G$ preserves a ray $l$ inside $L$, and since $q$ is nonzero on $l, G$ must fix $l$ pointwise. Since $G$ preserves $q$ and fixes $l$, it preserves another quadratic form $q^{\prime}$ which agrees with $q$ on $l^{\perp}$ and equals $-q$ on $l$. Since $q^{\prime}$ is positive definite, $G$ must be compact, a contradiction. Thus $\Lambda \subset \partial L$.

We prove 1.3(a) inductively on $n$. For $n=2, E(1,1)$ is already amenable so the closed subgroup $N(G)$ is automatically amenable. Assume that 1.3(a) has been proved for all dimensions less than $n$. We divide the proof into two cases, depending on whether $\Sigma \subset \partial L$. 
If $\Sigma \subset \partial L$, then $\operatorname{dim} \Sigma=1$. The stabilizer in $O(n-1,1)$ of such a $\Sigma$ is amenable (it is easily seen to be isomorphic to the Euclidean similarity group $\left.\left(\mathbf{R}^{*} \times O(n-2)\right) \ltimes \mathbf{R}^{n-2}\right)$ and since closed subgroups of amenable groups are amenable, $N(G)$ is amenable.

Otherwise $\Sigma \not \subset L$ and $L$ intersects $\Sigma$ in an open cone in $\Sigma$. Thus $\left.q\right|_{\Sigma}$ is indefinite and there exists $g \in O(n-1,1)$ such that $g(\Sigma)=\{0\} \times \mathbf{R}^{n-k} \subset \mathbf{R}^{n}$, $k=n-\operatorname{dim} \Sigma$. Then $g\left(\Sigma^{\perp}\right)=\mathbf{R}^{k} \times\{0\}$ and $N(G)$ preserves the orthogonal decomposition $\mathbf{R}^{n}=\Sigma^{\perp} \oplus \Sigma$. Hence $g^{-1} N(G) g \subset O(k) \times O(n-k-1,1)$. Since $G$ is noncompact it must project to a noncompact amenable subgroup of $O(n-k-1,1)$. Since $k>0, N(G)$ must project to a closed amenable subgroup of $O(n-k-1,1)$ by the induction hypothesis. It follows that $N(G)$ is amenable and (a) is proved.

Now suppose that $G$ is compact. For any $v \in L$ the orbit $G v$ is compact and its barycenter is a vector in $L$ fixed by $G$. Thus $\Lambda$ meets $L$ and $\Sigma$ is the span of $\Lambda \cap L$. Since $G$ fixes $\Lambda \cap L$ pointwise $G$ is the identity on $\Sigma$.

Suppose first that $\operatorname{dim} \Sigma \geqslant 2$. Then as in the preceding argument, there exists $g$ such that $g(\Sigma)=\{0\} \times \mathbf{R}^{n-k}, g\left(\Sigma^{\perp}\right)=\mathbf{R}^{k} \times\{0\}$ and $g^{-1} N(G) g \subset$ $O(k) \times O(n-k-1,1)$. As $G$ is the identity on $\Sigma$ it follows that $g^{-1} G g \subset$ $O(k) \times\{1\}$.

Now suppose $\operatorname{dim} \Sigma=1$. Then $G$ fixes a unique ray in $L$ and so must $N(G)$. It follows that $N(G)$ is compact.

The proof of 1.3 is now complete.

\section{Lorentz flat manifolds with discrete holonomy groups}

The purpose of this section is to prove the following

2.1. Proposition. Let $\Gamma \subset E(n-1,1)$ be a finitely generated subgroup which acts properly discontinuously on $\mathbf{R}^{n}$ with compact quotient. Assume that the image $L(\Gamma) \subset O(n-1,1)$ is discrete. Then $\Gamma$ is virtually polycyclic.

If $\Gamma \subset E(n-1,1)$ acts properly discontinuously on $\mathbf{R}^{n}$, then certainly $\Gamma$ is discrete, but the converse does not hold (e.g. no infinite discrete subgroup of $O(n-1,1) \subset E(n-1,1)$ acts properly discontinuously on $\left.\mathbf{R}^{n}\right)$. Similarly, if $\Gamma$ acts properly discontinuously on $\mathbf{R}^{n}$, then its image $L(\Gamma)$ under $L: E(n-1,1)$ $\rightarrow O(n-1,1)$ may or may not be a discrete subgroup of $O(n-1,1)$. In this section we consider the case that $L(\Gamma)$ is discrete in $O(n-1,1)$.

2.2. The proof proceeds by induction on the dimension $n$. For $n=1$ any such $\Gamma$ must either be cyclic on an infinite dihedral group $\mathbf{Z} \rtimes(\mathbf{Z} / 2)$, and there is nothing to prove. 
Assume the statement is true for all dimensions less than $n>1$. We show $\Gamma$ is virtually polycyclic. Since $\Gamma$ is a finitely generated subgroup of $E(n-1,1)$ $\subset \mathrm{GL}(n+1, \mathbf{R})$, it follows from Selberg's lemma (Raghunathan $[14,6.11])$ that $\Gamma$ contains a torsion-free subgroup of finite index. Replacing $\Gamma$ by a subgroup of finite index we may assume that $\Gamma$ is a torsion-free subgroup of $E(n-1,1)^{0}$.

The kernel of $L: \Gamma \rightarrow S O(n-1,1)$ is the subgroup $\Gamma \cap T$ of $\Gamma$ consisting of translations. Clearly, $\Gamma \cap T$ is a discrete group of translations. First we show that $\Gamma \cap T$ is nontrivial.

Suppose $\Gamma \cap T=\{0\}$, i.e. that $L: \Gamma \rightarrow L(\Gamma)$ is an isomorphism. Then the double coset space $L(\Gamma) \backslash S O(n-1,1) / O(n-1)$ is an $(n-1)$-dimensional hyperbolic space form and hence an aspherical $(n-1)$-manifold with fundamental group isomorphic to $\Gamma$. In particular the cohomological dimension of $\Gamma$ is at most $n-1$, contradicting the fact that $\Gamma$ is the fundamental group of the compact aspherical $n$-manifold $\mathbf{R}^{n} / \Gamma$.

2.3. Thus $T \cap \Gamma$ is nontrivial. Let $V$ be the subspace spanned by $T \cap \Gamma$; evidently $V$ is invariant under $L(\Gamma)$. Let $k=\operatorname{dim} V>0$ and let $b: V \times V \rightarrow \mathbf{R}$ be the Lorentzian bilinear form restricted to $V$. We distinguish three cases: (i) $b: V \times V \rightarrow \mathbf{R}$ is degenerate; (ii) $b: V \times V \rightarrow \mathbf{R}$ is positive definite (i.e. has signature $(k, 0)$; and (iii) $b: V \times V \rightarrow \mathbf{R}$ is nondegenerate but not positive definite (i.e. has signature $(k-1,1))$.

We start with case (i). Since $V$ is $L(\Gamma)$-invariant, it follows from 1.2 that $\Gamma$ must be virtually polycyclic.

2.4. For the other two cases (i) and (ii) consider the splitting of $\mathbf{R}^{n}$ as the orthogonal direct sum $\mathbf{R}^{n}=V \oplus V^{\perp}$. Let $O\left(V \oplus V^{\perp}\right)$ denote the subgroup of $O(n-1,1)$ which preserves $V$ (and hence the splitting $V \oplus V^{\perp}$ ). Then $O\left(V \oplus V^{\perp}\right)$ is a direct product $O(V) \times O\left(V^{\perp}\right)$, where $O(V)$ and $O\left(V^{\perp}\right)$ are the respective orthogonal groups of the quadratic forms restricted to $V$ and $V^{\perp}$. Let $E\left(V \oplus V^{\perp}\right)$ be the subgroup $E(n-1,1)$ generated by $\mathbf{R}^{*}$. $O\left(V \oplus V^{\perp}\right)$ and the group of translations $T$; clearly $E\left(V \oplus V^{\perp}\right)$ is the semidirect product $O\left(V \oplus V^{\perp}\right) \ltimes T$ and the direct product $E(V) \times E\left(V^{\perp}\right)$ where $E(V)$ (resp. $E\left(V^{\perp}\right)$ ) is the group generated by $O(V)$ (resp. $\left(V^{\perp}\right)$ ) and the translations in $V$ (resp. $\left.V^{\perp}\right)$. Let $p: E\left(V \oplus V^{\perp}\right) \rightarrow E\left(V^{\perp}\right)$ denote the canonical projection.

By another application of Selberg's lemma we may replace $\Gamma$ by a subgroup of finite index in order to assume the image of $\Gamma$ under $E\left(V \oplus V^{\perp}\right) \rightarrow O(V)$ is torsion free.

We may consider the covering space $\hat{M}=\mathbf{R}^{n} /(\Gamma \cap T)$ of $M$. The action of $V$ on $\mathbf{R}^{n}$ by translations defines an action of the $k$-torus $(k=\operatorname{dim} V), T^{k}=$ $V /(\Gamma \cap T)$ on $\hat{M}$. Indeed, $\hat{M}$ becomes a principal $T^{k}$-bundle over $V^{\perp}$. 
2.5. Lemma. $\Gamma \cdot V$ acts properly on $\mathbf{R}^{n}$ and $p(\Gamma)$ acts properly discontinuously on $V^{\perp}$.

Recall that a group $G$ acts properly on a space $X$ if the canonical mapping $G \times X \rightarrow X \times X$ defined by $(g, x) \mapsto(g x, x)$ is proper. A group acts properly discontinuously if and only if it acts properly when it is given the discrete topology. We must show that for any two compact subsets $K_{1}, K_{2} \subset \mathbf{R}^{n}$ the set of all $v \gamma \in V \cdot \Gamma$ such that $\gamma v K_{1}$ meets $K_{2}$ is compact. To this end let $F \subset V$ be a compact subset which meets each $\Gamma \cap T$-coset. $\left\{v \gamma \in V \cdot \Gamma \mid v \gamma K_{1} \cap K_{2} \neq \varnothing\right\}$ is a closed subset of the set $F \cdot\left\{\gamma \in \Gamma \mid \gamma K_{1} \cap K_{2} \neq \varnothing\right\}$, which is compact since $\Gamma$ acts properly. It follows that $\Gamma \cdot V=V \cdot \Gamma$ acts properly. Since $p(V)$ is trivial and $\Gamma$ is discrete in $E\left(V \oplus V^{\perp}\right)$, it follows that $P(\Gamma)$ is discrete in $E\left(V^{\perp}\right)$ and acts properly discontinously on $V^{\perp}$. The proof of Lemma 2.5 is complete.

2.6. Next we show that the kernel of $p: \Gamma \rightarrow E\left(V^{\perp}\right)$ is precisely $\Gamma \cap \mathbf{R}^{n}=$ $\operatorname{Ker} L: \Gamma \rightarrow O(n-1,1)$. For if $\gamma \in \Gamma, p(\gamma)=1$ implies that $\gamma$ acts as the identity on $V^{\perp}$. Thus the induced action of $\gamma$ on $\hat{M}$ preserves each $T^{k}$-fiber of $\hat{M} \rightarrow V^{\perp}$. Since $\gamma$ acts properly discontinuously on $\hat{M}$, it must act properly discontinuously on each $T^{k}$ and hence its linear part is restricted to $V .\left.L(\gamma)\right|_{V}$ must have finite order. But this implies $L(\gamma)=1$ and hence $\gamma$ is a translation.

We have thus proved: there is an exact sequence

$$
\mathbf{Z}^{k} \rightarrow \Gamma \stackrel{p}{\rightarrow} p(\Gamma)
$$

and $p(\Gamma)$ acts properly discontinuously on $V^{\perp}$. In particular, $\Gamma$ is virtually polycyclic if and only if $p(\Gamma)$ is.

2.7. We conclude the proof of 2.1 in case (i), when the Lorentz metric on $\mathbf{R}^{n}$ restricts to a positive definite form on $V$. Then $V^{\perp}$ has a $p(\Gamma)$-invariant flat Lorentz structure and $V / p(\Gamma)$ is compact. Since $\operatorname{dim} V^{\perp}<n$, it follows from the induction hypothesis that $p(\Gamma)$, and hence $\Gamma$, is virtually polycyclic.

2.8. Finally we consider case (ii): when $V$ inherits a nondegenerate-but nonpositive-form from $\mathbf{R}^{n}$. In that case $p(\Gamma)$ acts on $V^{\perp}$ by Euclidean isometries (for some Euclidean metric on $V^{\perp}$ ). It follows from the classical Bieberbach theorems (see e.g. Wolf [15, 3.2.1] or Auslander [1]) that such a group is virtually abelian. Therefore, $\Gamma$ is virtually polycyclic and the proof of 2.1 is complete.

\section{Complete Lorentz flat manifolds with indiscrete linear holonomy group}

The purpose of this section is to complete the proof of virtual polycyclicity of the fundamental group of a compact flat Lorentz space form. 
3.1. Theorem. Let $\Gamma \subset E(n-1,1)$ act properly discontinuously with $\mathbf{R}^{n}$ a compact quotient. Suppose that the linear holonomy group $L(\Gamma) \subset O(n-1,1)$ is not discrete. Then $\Gamma$ is virtually polycyclic.

3.2. One of our principal tools is the following, for which a discussion may be found in Raghunathan [14, 8.24].

Theorem. (Auslander [2]). Let $\Gamma$ be a discrete subgroup of a semidirect product $G=A \rtimes B$, where $A$ is a solvable normal subgroup. Let $\rho: G \rightarrow B$ be the canonical projection with kernel $A$. Then the identity component $\overline{\rho(\Gamma)^{0}}$ of the closure of $\rho(\Gamma)$ is solvable.

Using this fact we can now obtain

3.3. Proposition. Suppose $\Gamma \subset E(n-1,1)$ is a discrete subgroup and the identity component $\overline{L(\Gamma)^{0}}$ of the linear part $L(\Gamma) \subset O(n-1,1)$ is noncompact. Then $\Gamma$ is virtually polycyclic.

Proof. By Auslander's theorem $G=\overline{L(\Gamma)}^{0}$ is a connected closed solvable subgroup of $O(n-1,1)$. Furthermore, $L(\Gamma)$ normalizes $G$. By hypothesis, $G$ is noncompact and by 1.3(a) $L(\Gamma)$ lies in the amenable subgroup $N(G)$ of $O(n-1,1)$. Thus $\Gamma$ is a discrete subgroup of the Zariski-closed amenable group $N(G) \ltimes T \subset E(n-1,1)$ and by $1.1 \Gamma$ is virtually polycyclic. q.e.d.

3.4. Thus we know that a subgroup $\Gamma \subset E(n-1,1)$ which acts properly discontinuously on $\mathbf{R}^{n}$ with compact quotient is virtually polycyclic if either (i) $L(\Gamma)$ is discrete in $O(n-1,1)$ or (ii) the identity component of the closure of $L(\Gamma)$ is noncompact. We finish the proof of Theorem 3.1, and in the last remaining case: under the assumption that $\overline{L(\Gamma)}^{0}$ is compact.

By $2.2, G=\overline{L(\Gamma)}^{0}$ is solvable and in fact abelian (since it is also compact and connected). By Lemma 1.3(b), either $N(S)$ is compact or $N(S)$ is conjugate to a subgroup of $O(k) \times O(n-k-1,1)$ in such a way that $S$ maps to $O(k)$. In the first case $\Gamma$ lies in the amenable group $N(S) \ltimes T$ and must be virtually polycyclic. Thus we may assume that $S \subset O(k) \times\{1\}$ and $L(\Gamma) \subset$ $N(S) \subset O(k) \times O(n-k-1,1)$.

Let $\rho$ denote the composition

$$
\Gamma \stackrel{L}{\rightarrow} L(\Gamma) \subset O(k) \times O(n-k-1,1) \rightarrow O(n-k-1,1),
$$

and $\Gamma_{1}$ denote its kernel. Then $\Gamma_{1}$ is a properly discontinuous group of Euclidean isometries; passing to a subgroup of finite index in $\Gamma$ we may assume $\Gamma$ (and hence $\Gamma_{1}$ ) acts freely on $\mathbf{R}^{n}$. Thus $\mathbf{R}^{n} / \Gamma_{1}$ is a complete flat Riemannian manifold. By Wolf $[15,3.3 .3], \mathbf{R}^{n} / \Gamma_{1}$ deformation retracts onto a compact totally geodesic submanifold, which must be of the form $F / \Gamma_{1}$, where $F$ is a $\Gamma_{1}$-invariant affine subspace. Moreover, all the $\Gamma_{1}$-invariant affine subspaces $F$ such that $F / \Gamma_{1}$ is compact are all parallel and their union is itself 
an affine subspace $E$ of $\mathbf{R}^{n}$. Since $\Gamma_{1}$ is normal in $\Gamma$, the various $F$ are permuted by $\Gamma$ and $E$ is a $\Gamma$-invariant affine subspace. By Fried, Goldman \& Hirsch [9, 2.1], $E=\mathbf{R}^{n}$.

Thus we may choose one $F \subset \mathbf{R}^{n}$ upon which $\Gamma_{1}$ acts as a Euclidean crystallographic group; then $\Gamma_{1}$ acts as a Euclidean crystallographic group on each coset $x+F, x \in \mathbf{R}^{n}$. It follows that all of the eigenvalues of elements of $L\left(\Gamma_{1}\right)$ are roots of unity. By Selberg's lemma, we may replace $\Gamma$ by a subgroup of finite index none of whose elements have eigenvalues which are roots of unity except 1 . Thus we may assume that $\Gamma_{1}$ consists of translations.

We claim now that $L(\Gamma)$ must be discrete. Since $S \subset O(k) \times\{1\}$, the image $\rho(\Gamma)$ is discrete in $O(n-k-1,1)$, i.e. $L(\Gamma) \subset O(k) \times O(n-k-1,1)$ projects to a discrete subgroup of $O(n-k-1,1)$. Since $L(\Gamma)=1$ whenever $\rho(\gamma)=1$, it follows that $L(\Gamma)$ is discrete. By $1.1, \Gamma$ must be virtually polycyclic.

\section{References}

[1] L. Auslander, An account of the theory of crystallographic groups, Proc. Amer. Math. Soc. 11 (1960) 687-688.

[2] _ On radicals of discrete subgroups of Lie groups, Amer. J. Math. 85 (1963) 145-150.

[3] L. Auslander \& L. Markus, Holonomy of flat affinely connected manifolds, Ann. of Math. 82 (1955) 139-151.

[4] _ Flat Lorentz 3-manifolds, Mem. Amer. Math. Soc., 1959.

[5] E. Calabi \& L. Markus, Relativistic space forms, Ann. of Math. 75 (1962) 63-76.

[6] S. S. Chen \& L. Greenberg, Hyperbolic spaces, Contributions to Analysis, Academic Press, New York, 1974, 48-87.

[7] D. Fried, in preparation.

[8] D. Fried \& W. Goldman, Three-dimensional affine crystallographic groups, Advances in Math. 48 (1983) 1-49.

[9] D. Fried, W. Goldman \& M. W. Hirsch, Affine manifolds with nilpotent holonomy, Comment Math. Helv. 56 (1981) 487-523.

[10] L. Greenberg, Discrete subgroups of the Lorentz group, Math. Scand. 10 (1982) 85-107.

[11] F. Greenleaf, Invariant means on topological groups, Van Nostrand Math. Series 16, 1969.

[12] Y. Kamishima, Properly discontinuous actions of subgroups in amenable algebraic groups and its application to affine motions, Topology and its Applications, to appear.

[13] J. Milnor, On fundamental groups of complete affinely flat manifolds, Advances in Math. 25 (1977) 178-187.

[14] M. S. Raghunathan, Discrete subgroups of Lie groups, Ergebnisse band 68, Springer, Berlin and New York, 1972.

[15] J. A. Wolf, Spaces of constant curvature, McGraw-Hill, New York, 1967, and Publish or Perish, Boston, 1974.

Massachusetts Institute of Technology HOKKAIDO UNIVERSITY 